\title{
Hydrodynamic Analysis of Binary Immiscible Metallurgical Flow in a Novel Mixing Process - Rheomixing
}

\author{
H. Tang ${ }^{1,2, *}$, L.C. Wrobel ${ }^{1}$, Z. Fan ${ }^{2}$ \\ ${ }^{1}$ Department of Mechanical Engineering, Brunel University, Uxbridge, Middlesex, UB8 3PH, UK \\ ${ }^{2}$ Brunel Centre for Advanced Solidification Technology, Brunel University, Uxbridge, Middlesex, UB8 3PH, UK
}

\begin{abstract}
This paper presents a hydrodynamic analysis of binary immiscible metallurgical flow by a numerical simulation of the rheomixing process. The concept of multi-controll is proposed for classifying complex processes and identifying individual processes in an immiscible alloy system in order to perform simulations. A brief review of fabrication methods for immiscible alloys is given, and fluid flow aspects of a novel fabrication method - rheomixing by twin-screw extruder (TSE) are analysed. Fundamental hydrodynamic micro-mechanisms in a TSE are simulated by a piecewise linear (PLIC) volume-of-fluid (VOF) method coupled with the continuum surface force (CFS) algorithm. This revealed that continuous reorientation in the TSE process could produce fine droplets and the best mixing efficiency. It is verified that TSE is a better mixing device than single screw extruder (SSE) and can achieve finer droplets. Numerical results show good qualitative agreement with experimental results. It is concluded that rheomixing by a TSE can be successfully employed for casting immiscible engineering alloys due to its unique characteristics of reorientation and surface renewal.
\end{abstract}

PACS: 47.11.+j; 68.05.Gh; 83.50.Xa 


\section{Introduction}

Some alloys exhibit two immiscible liquid phases within a certain temperature range. These are advanced materials suitable for superconducting devices, electrical contacts, and bearing materials if the soft phase A is well dispersed in the solid matrix phase B [1]. However, the different densities of these liquid phases lead, on Earth, to the formation of two layers. This prevents the homogeneous distribution of particles in a matrix using simple and inexpensive casting processes. Therefore, the industrial exploitation of such alloys has also been limited so far. Immiscible alloys have been studied intensively for the last decades, mainly focusing on two aspects: the fundamental mechanism of secondary phase segregation/coarsening; and mixing approaches for the fabrication process. Amongst these, the hydrodynamic behaviour of liquid immiscible metallic alloy flows is important. The flow field of liquid immiscible alloys includes phenomena such as nucleation, coarsening and diffusion, Marangoni and Stokes motions, collision and coagulation. The phase diagram of the immiscible alloy system coupled with the operating conditions, consisting of multicontrolled processes, determine the flow features. Possible considerations for the numerical simulation of the twin-screw rheomixing process will be discussed in section 2.2.

Evidence shows that the solidified microstructure of cast immiscible alloys strongly depends on the hydrodynamic behaviour of the liquids during cooling [2]. Therefore, there is an increasing need to be able to control these complex metallurgical processes and hence, an improved capability to numerically simulate and study these processes. Numerical simulations are, in principle, ideally suited to study these complex immiscible interfacial flows and to provide an insight into the process that is difficult to obtain by experimentation.

The hydrodynamic analysis of binary immiscible liquid alloys by a numerical evaluation presented here involves simplified problems that reflect the main flow features in the rheomixing process. The concept of multi-controlled process is proposed for classifying complex processes in an immiscible alloys system and identifying individual processes. A brief review of fabrication methods for immiscible alloys is given and fluid flow aspects of a novel fabrication method - rheomixing by a twin-screw extruder are analysed. Fundamental hydrodynamic micro-mechanisms in 
rheomixing process are simulated by a piecewise linear (PLIC) [3] volume-of-fluid (VOF) method [4] coupled with the continuum surface force (CFS) algorithm [5].

\section{Binary immiscible liquid alloys system}

\subsection{Rheological and thermodynamic behaviour}

A miscible gap in the liquid state is found in metal-metal systems such as Al-Bi, $\mathrm{Al}-\mathrm{Pb}, \mathrm{Cu}-\mathrm{Pb}, \mathrm{Zn}-\mathrm{Pb}, \mathrm{Ga}-\mathrm{Pb}$ etc. A typical equilibrium phase diagram is shown in Fig.1. The flow behaviour of the binary system is generally determined by its phase diagram. Above the so-called critical temperature $T_{\mathrm{c}}$, both components are completely miscible to every amount or proportion in the liquid state. Below $T_{\mathrm{c}}$, two liquids of different compositions, $L_{1}$ and $L_{2}$, coexist in equilibrium as a two-phase liquid state. At $T_{\text {mon }}$, liquid $L_{1}$ is no longer stable and a monotectic reaction occurs, while a solid phase $S_{\mathrm{A}}$ appears in liquid phase $L_{2}$. The rheological behaviour of $L_{1}+L_{2}$ is significant and will affect the microstructure of solidification in the final casting, which will also be influenced by different fabrication processes [6].

The recent progress in liquid immiscible alloys has been reviewed by Ratke and Diefenbach [2]. Zeng et al. [7] presented the computerized prediction of phase diagrams of liquid-liquid immiscible binary systems between non-transition elements. Good agreement is reached for a Ga-Pb binary system. Zhang et al. [8] reported a calculation that was carried out numerically for an immiscible phase in the $\mathrm{Zn}-\mathrm{Pb}$ binary alloy system. The agreement between the calculated and experimentally determined values of activity coefficient is excellent, indicating the validity of the NRTL equation model for the description of immiscible solutions. Plevachuk et al. [9] performed electroconductivity measurements for binary and ternary immiscible In-Se, In-Te, Tl-Se, Tl-Te and Tl-Se-Te system alloys. Shim et al. [10] studied the liquid miscibility gap in the $\mathrm{Al}-\mathrm{Pb}-\mathrm{Sn}$ system, and reported that the liquid miscibility gap boundary of two immiscible liquids, $\mathrm{Al}$ - and $\mathrm{Pb}$-rich, has been measured in a temperature range between 1073 and $1293 \mathrm{~K}$. A set of ternary liquid thermodynamic parameters has been evaluated based on experimental data measured in that work, together with phase equilibrium data available in the literature. From these, the phase diagram of the $\mathrm{Al}-\mathrm{Pb}-\mathrm{Sn}$ system, including the liquid miscibility gap, has been 
calculated using the CALPHAD method. The calculated results are in good agreement with experimental data. A review of phase diagram principles and computations has been given in [11]. Smith and Elmer [12], Gueneau et al. [13] have also presented studies of immiscible alloys. Ratke and Alkemper [14] worked on modelling of phase separation in liquids with a miscibility gap.

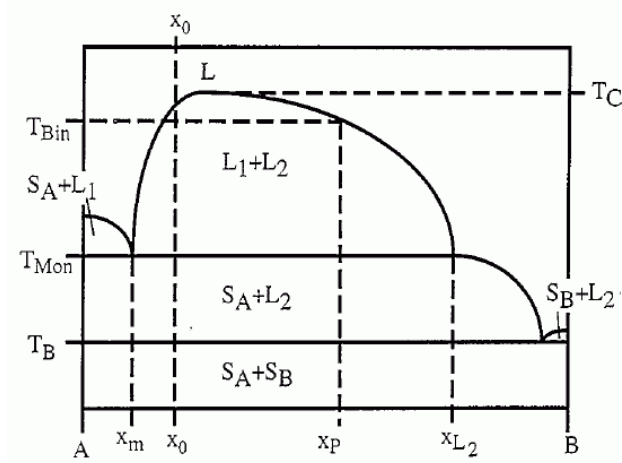

Fig. 1 Typical binary phase diagram with a miscible gap in the liquid state

\subsection{The concept of multi-controlled process}

The flow fields of liquid immiscible metallic alloys under conventional processes include the effects of nucleation, coarsening and diffusion, Marangoni and Stokes motions, collision and coagulation. However, by imposing an external mobile source (see Fig.2), such as mechanical stirring, enhanced micro- and macro-structural flow fields are induced, which dominate the flow features. Possible considerations are depicted in Fig. 2. The concept of multi-controlled processes is developed comparing with conventional processes as shown in Fig. 2. The object is to classify and identify flow regions of immiscible liquid alloys, and determine the dominant flow in each process that can be studied numerically for charactering the fundamental mechanisms of rheomixing by TSE process. The important parameters or functions are shown in Fig. 2, below the name of each process, where $\Delta G_{V}$-volume Gibbs free energy, $\sigma$ interface tension, $D$-diffusion coefficient, $\frac{\partial \sigma}{\partial T}$-temperature-dependent interface tension, $\nabla T$-temperature gradient, $\eta$-shear viscosity, $\lambda$-mean distance, $\rho$-density, ReReynolds number, $\dot{\gamma}$-shear rate, $\delta$-flow channel width. The numerical studies of the microstructural mechanisms should comprehensively involve different numerical 
approaches and modelling at each stage, according to which fluid phenomena play a dominant role.

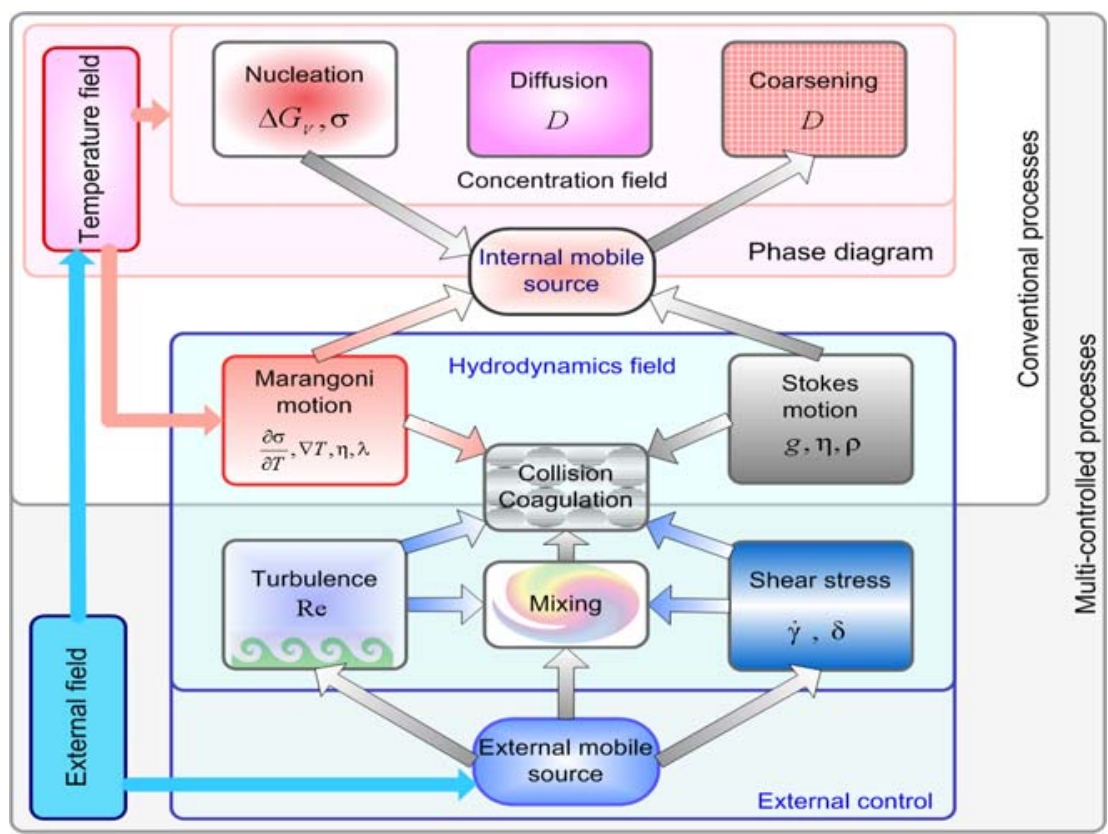

Fig.2 Sketch of the interdependence of conventional processes and multi-controlled processes for the numerical simulation of immiscible liquid alloys

\subsection{Dominating flow features}

Marangoni and Stokes motions will dominate the flow features of immiscible casting processes $[6,15]$, and will significantly affect the dispersion and distribution of the drop phase on the quality of final casting. The investigation of Marangoni and Stokes motions in the fabrication process is interesting, as it reveals the microstructure mechanism of stability of the mixed phase flow.

The velocity of a drop caused by thermocapillary or Marangoni motion (Marangoni convection) can be described by one of the following equations:

$$
U_{m}=\frac{2}{\eta_{p}(2-\hat{\lambda})(2+2 \hat{\eta})} \frac{d \sigma}{d T} \nabla T R
$$

where $d \sigma / d T$ is the temperature dependence of the interfacial tension, $\hat{\lambda}$ is the ratio of the thermal conductivity of the droplet to the matrix, $\nabla T$ is the temperature gradient, and $\hat{\eta}$ is the ratio of the viscosity of the droplet to the matrix [2], or 


$$
\begin{aligned}
& U_{m}=\frac{2\left|\frac{d T}{d x}\right|\left|\frac{d \sigma_{m d}}{d T}\right| \kappa_{m}}{\left(2 \kappa_{m}+\kappa_{d}\right)\left(2 \eta_{m}+3 \eta_{d}\right)} r \\
& U_{m}=\frac{2 k_{l} \nabla \sigma}{\left(2 k_{1}+k\right)\left(2 \eta_{1}+3 \eta_{2}\right)} r
\end{aligned}
$$

where $k_{1}$ and $k_{2}$ are the thermal conductivity of the matrix and dispersion phases, respectively, $\nabla \sigma$ is the surface tension gradient, $\eta_{1}$ and $\eta_{2}$ represent the viscosity of the droplet and matrix respectively [16], or finally [17]

$$
U_{m}=-\left[\frac{2}{3 \eta_{2}\left(2 \eta_{2}+3 \eta_{1}\right)}\right]\left[\frac{3 \eta_{2} r}{\left(2+h^{\prime} / h\right)}\left(\frac{\partial \sigma}{\partial T}\right) \nabla T+\left(\rho_{2}+\rho_{1}\right)\left(\eta_{2}-\eta_{1}\right) g r^{2}\right]
$$

The Marangoni number $\mathrm{M}$ is defined as:

$$
\mathrm{M}=\frac{\left|\sigma_{T}\right| \Delta T L}{\mu \alpha}
$$

where $\sigma_{T}$ is the temperature gradient of surface tension, $\Delta T$ the temperature difference between hot and cold disks, $L$ a characteristic length of the fluid, $\mu$ dynamic viscosity, and $\alpha$ thermal conductivity. Alternative expressions are as follows:

$$
\begin{aligned}
& \mathrm{M}=\frac{|(\partial \sigma / \partial T) \Delta T| r^{2}}{\mu \alpha} \\
\text { or } \quad & \mathrm{M}=\frac{\sigma \beta|\nabla T| r}{\rho v^{2}}
\end{aligned}
$$

where $\beta=$ constant $>0$ [18].

The Stokes motion of a droplet in the matrix phase can be described by [2]:

or

$$
\begin{aligned}
& U_{s}=\frac{9}{2} \frac{\Delta \rho}{\eta_{m}} \frac{1+\hat{\mu}}{1 / 3+\hat{\mu}} g r^{2} \\
& U_{s}=\frac{2}{3} \frac{\left(\rho_{2}-\rho_{1}\right)\left(\eta_{2}+\eta_{1}\right)}{\eta_{1}\left(3 \eta_{2}+2 \eta_{1}\right)} g r^{2}
\end{aligned}
$$

Equation (9) is a well-known formula derived by the Hadamard-Rybczynski theory [18].

Lu et al. [19] investigated the microstructure of an iron-copper binary system by using equations (4) and (9). Nestler et al. [20] studied the Marangoni flow in a binary monotectic alloy system by the phase-field method. Rudraiah et al. [21], Hibiya et al. [22], Takagi et al. [23] studied Marangoni flow by using equation (5). A Marangoni phenomenon has been investigated in space $[17,24,25]$, because the Stokes motion 
dominated the flow features on Earth. Rhazi et al. [26] presented their results for the stability of free convection in a particle-fluid mixture with a free surface, using equation (6) as one of the evaluating parameters. Rakte et al. ([27] investigated droplet Marangoni motion in immiscible Al-Si-Bi alloys in space.

Marangoni migration is caused by the surface tension gradient of the dispersion droplets in the matrix phase, which is related to the gradients of concentration and temperature. The droplet tends to be moved in the direction of decreasing surface tension. Marangoni is a surface-driven convection finally depending on the gradients of temperature. Marangoni only occurs in thin laminar flow. The discussion of convection effects can be described by three dimensionless numbers:

$\begin{array}{lr}\text { Peclet number } & \mathrm{Pe}=\frac{U r}{D} \\ \text { Schmidt number } & \mathrm{Sc}=\frac{v}{D}\end{array}$

Reynolds number $\quad \operatorname{Re}=\frac{U r}{v}$

where $U$ is the particle velocity, $v$ is the kinematic viscosity, $r$ is the diameter of the particle, and $D$ is the diffusion coefficient.

The Peclet number determines whether solute transport occurs mainly by convection $(\mathrm{Pe}>>1)$ or by diffusion $(\mathrm{Pe}<<1)$. The Schmidt number characterises the amount of momentum transfer, expressed by the kinematic viscosity in relation to that of diffusional momentum transfer. For metallic liquids, the Schmidt number is of the order Sc $\approx 100$. This means that the thermal field relaxes about 100 times faster than the solute field. The Reynolds number divides the regimes of creeping flow $(\operatorname{Re}<1)$ and turbulent flow $(\operatorname{Re}>>1)$ [2]. The normal approach for an immiscible liquid metal flow is to consider it as a creeping, laminar flow in a viscous Newtonian liquid.

\section{Fabrication processes}

A number of attempts have been made to obtain a homogeneous distribution for various immiscible alloys, including stirring, rapid/directional solidification, ultrasonic/magnetic vibration, powder metallurgy, and microgravity. In microgravity, model materials also unexpectedly yielded separated phases. This is attributed to a 
droplet migration mechanism (Marangoni migration), which is driven by gradients of interfacial tension along the surfaces of droplets. These gradients relate directly to the local temperature gradients in the matrix during cooling and solidification and are, therefore, independent of gravity. With the results from microgravity experiments, this mechanism was studied on a theoretical basis and models were developed. The possibility of controlling the direction of migration and the velocity of droplets through mastering the heat flow in molten alloys has been demonstrated. The ideal situation would be to minimise the sedimentation caused by gravity by balancing it with the Marangoni migration effect. If droplets reach a state of dynamic equilibrium, then a stable dispersion in a solidifying matrix becomes attainable. This concept has been tested and new materials, for example an aluminium alloy matrix with evenlydistributed inclusions of lead or bismuth, are being tested as candidates for advanced sliding bearing in car engines [6]. Unfortunately, the Marangoni migration effect cannot be eliminated by counterbalancing the sedimentation due to orientation of temperature gradient [15]. However, the TSE rheomixing approach, invented at BCAST (Brunel Centre for Advanced Solidification Technology) by Fan et al. [1], delivers a novel process concept to solve these problems for industrial applications. Results show that the desired microstructure was achieved by the TSE process, with fine and uniform droplets and homogeneous dispersion and stable suspension in semisolid metal (SSM) state for advanced sliding bearings in car engines.

In these materials the hard matrix sustains the dynamic loads from the combustion process, while the soft inclusions provide self-lubrication. Developing this new fabrication method into an economically viable casting process would permit the development of more efficient engines.

\subsection{Fabrication processes}

Mixing two fluids consists generally of two successive processes. First, it is necessary to break up the minor fluid particles into smaller ones; this is called "intensive mixing". Then, to obtain a good spatial distribution, one spreads these particles within the matrix; this is called "distributive mixing". These two processes may be achieved simultaneously or stepwise. Since the beginning of the 1980s, studies of mixing focused on low viscosity materials for which the value of the Reynolds number was a key point: inducing turbulence was the best way to promote 
blending. However, for mixing highly viscous materials, the best way to promote mixing is to induce chaotic motion [28]. Various mixing devices were developed and categorised as dynamic or static mixers: internal mixers, single-screw extruder, twinscrew extruder, calendar, batch mixer, cams mixer, etc [29]. It is clear that efficient mixing involves two aspects: folding and reorienting [30].

The casting of immiscible alloys by conventional methods does not yield materials with the expected homogeneous particle distribution. Various fabrication methods [2, 31] for continuous or component processes were developed for experimental research and industrial applications, including powder metal, directional solidification, microgravity, stirring, orientating temperature gradient strip casting, rapid solidification and rheomixing $[32,33]$. Table 1 shows an overview of principles and problems for the main fabrication methods.

Table 1.

Overview of fabrication methods for immiscible alloys [1,2,31]

\begin{tabular}{lll}
\hline Fabrication & Principles & Problems \\
\hline Powder metal method & well mixed powder & complex, powder oxide \\
\hline Directional solidification & aligned monotectic growth & difficult to control, expensive \\
\hline Space station & eliminate $g$ & reduce $g$ \\
\hline
\end{tabular}




\subsection{A novel process-rheomixing by a twin screw extruder (TSE)}

Research in recent decades has shown that a fine dispersion of the minority phase particles in a hypermonotectic alloy can be obtained by rapid quenching or fast cooling, thus passing through the miscibility gap within a second or less. However, results show that the soft phase drops concentrate in the middle of the strip due to Marangoni motion [15]. The processing starts from a temperature above $T_{\mathrm{c}}$, which is not practical for industrial applications.

Rheomixing by a TSE extruder with specially designed screw profiles [1] shown in Fig. 3 is a highly efficient process to produce fine droplets and a homogeneous distribution. The main characteristics of the rheomixing process are:

- Size of particle: fine and uniform diameter.

- Morphology of particle: close to spherical shape.

- Dispersion of particle: homogeneous distribution.

- Suspension of particle: stable state for solidification

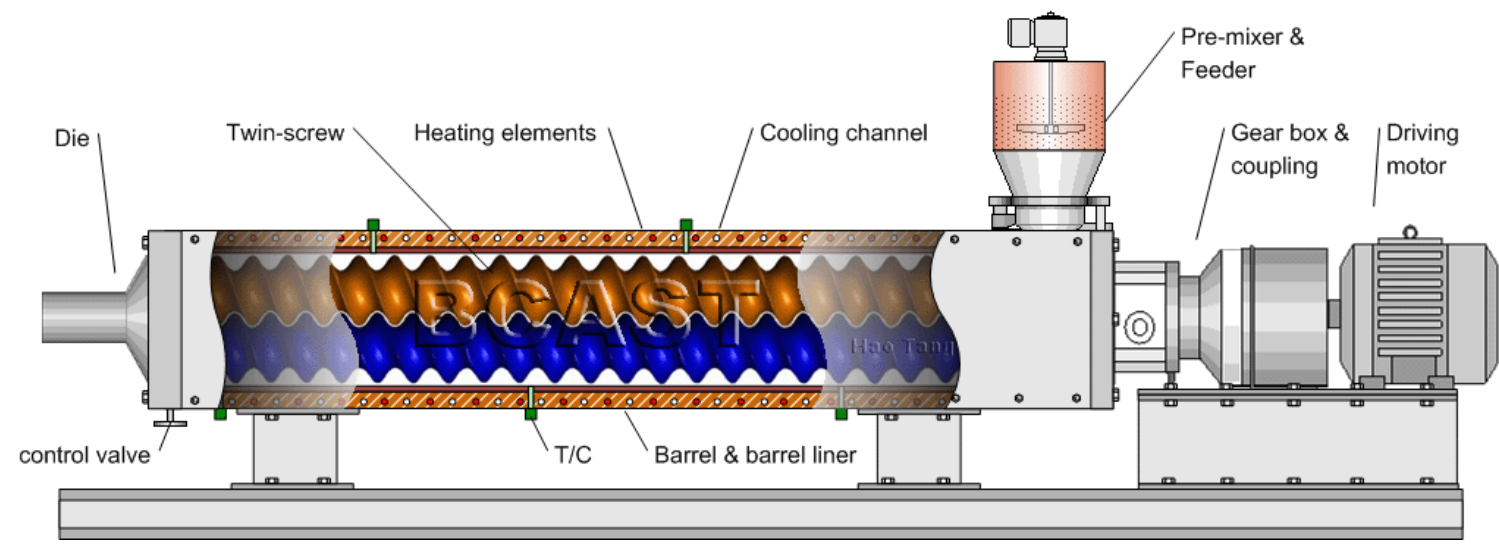

Fig.3 Schematic illustration of a TSE machine for rheomixing

Comparing existing fabrication processes as listed in Table 1, it can be seen that the rheomixing process has excellent advantages, including near-net shape production, one-step production, microstructural management, improved production efficiency, improved property combinations, lower overall cost.

Basically, the main feature of a twin-screw extruder is a strong shear flow field produced by co-rotating intermeshing screws $[34,35,36]$. Droplets are created in the microscopic scale, and by the turbulent flow enhanced by mixing, swirling and pumping actions in a macroscopic scale. The flow inside the rheomixing process is 
illustrated in Fig.4. Two immiscible liquid alloys are stirred before pouring into a twin-screw extruder, and the minority phase A liquid is in the form of larger droplets. The main concern for the rheomixing process is droplet interaction, including rupturing, coalescence and suspension, which affect the microstructure of immiscible alloy castings.

The study of immiscible binary alloys in shear-induced turbulent flows is important to provide more detailed information into the rheomixing process, to increase our control of the rheology of an emulsion and its solidified microstructure. An understanding of the fluid dynamics inside and around a suspended drop is necessary for delineating the mechanisms of microscopic transport and microstructure of immiscible alloys.

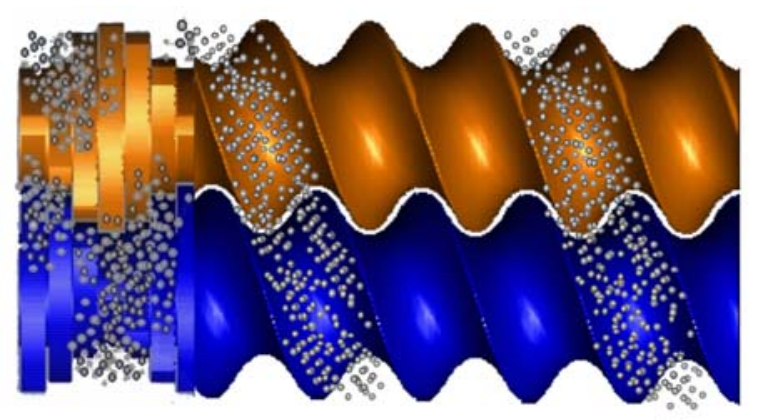

Fig. 4 Schematic illustration of flows inside TSE machine

\section{Numerical methods}

As described in previous sections, efforts have been made to understand the processes of immiscible alloys by simulation approaches. However, there is no approach that can cover all factors listed in Fig.2, from regions above $T_{\mathrm{c}}$ down to $T_{\mathrm{m}}$ in the phase diagram. Each approach can predict some of them while neglecting others. Most approaches have been developed for immiscible fluids in aerosol and other chemical areas, and further developments are needed to match the physical features of immiscible liquid alloys. Generally speaking, these approaches are powerful techniques to predict the decomposition and microstructural evolution, if the real thermophysical properties are known with enough accuracy, or alternatively, these approaches can be tested if these properties would be known accurately. Unfortunately, there is obviously a lack of knowledge on immiscible alloys, since 
interfacial tension, viscosity, diffusion coefficients are functions of composition, temperature, and shear rate for semisolid metals and not know with sufficient accuracy.

\subsection{Numerical approaches}

Immiscible metallic alloy flows are considered here as multi-phase fluid systems in isothermal state, with different density and viscosity. The domain of interest contains an unknown free boundary, which undergoes severe deformation and separation. The numerical methods adopted in the present simulations are based on Hirt and Nichols' VOF method [4] coupled with Youngs' piecewise linear interface construction (PLIC) scheme [3], Brackbills' continuum surface force (CSF) model [5], and solved by an algebraic multigrid (AMG) solver [37], as well as the $k-\varepsilon$ turbulence model [38] and the pressure-implicit with splitting of operators (PISO) scheme for pressure-velocity coupling [39]. A brief summary of the PLIC-VOF methodology is described as follows, detailed derivations are provided in the original literature cited above.

\subsection{VOF method}

The principal steps of VOF methods are reconstructed interface geometry and time integration algorithms. There are mainly three algorithms (Piecewise constant (SLIC), Piecewise constant stair-stepped (D-A), and Piecewise linear (PLIC)) for the reconstruction interface geometry and two algorithms (one dimensional or operator split and multidimensional or unsplit) for time integration.

The piecewise linear method is different from piecewise constant in that it reconstructs interface lines with a slope, which is given by the interface normal. The interface normal is determined with a multidimensional algorithm that does not rely on the sweep direction. PLIC volume tracking methods have been used successfully for capturing sharp fluid interfaces. [40] [41]. 


\subsubsection{The equation of motion}

The motion of the interface between two immiscible liquids of different density and viscosity in the VOF method is defined by a volume fraction function $C$. The following three conditions are possible:

$C=0 \quad$ fluid 1 , the cell is empty of fluid 2

$C=1 \quad$ fluid 2 , the cell is empty of fluid 1

$0<C<1$ the cell contains the interface between the two fluids

Based on the local value of $C$, the appropriate properties and variables are assigned to each control volume within the domain.

The volume fraction function $C$ is governed by the volume fraction equation

$$
\frac{\partial C}{\partial t}+\mathbf{u} \cdot \nabla C=0
$$

where $\mathbf{u}$ is the velocity of the flow.

The flow is governed by a single momentum equation, as shown below, with the resulting velocity field shared among the phases

$$
\rho\left(\frac{\partial \mathbf{u}}{\partial t}+\mathbf{u} \cdot \nabla \mathbf{u}\right)=-\nabla p+\mu \nabla^{2} \mathbf{u}+\rho g+F
$$

where $F$ stand for body forces, and $g$ for gravity acceleration.

The properties appearing in the momentum equation are determined by the presence of the component phase in each control volume, that is, the average value of density and viscosity are interpolated by the following formulas

$$
\begin{aligned}
& \rho=C_{\mathrm{m}} \rho_{\mathrm{m}}+\left(1-C_{\mathrm{m}}\right) \rho_{\mathrm{d}} \\
& \mu=C_{\mathrm{m}} \mu_{\mathrm{m}}+\left(1-C_{\mathrm{m}}\right) \mu_{\mathrm{d}}
\end{aligned}
$$

\subsubsection{Geometric reconstruction scheme for the VOF method}

The formulation of the VOF model requires that the convection and diffusion fluxes through the control volume faces be computed and balanced with source terms within the cell itself. The interface will be approximately reconstructed in each cell by a proper interpolating formulation, since we lose interface information when the interface is represented by a volume fraction field.

The geometric reconstruction PLIC scheme (piecewise linear interface construction) is employed because of its accuracy and applicability for general unstructured meshes, compared to other methods such as the donor-acceptor, SLIC, 
Euler explicit, and implicit schemes. A VOF geometric reconstruction scheme is divided into two parts: a reconstruction step and a propagation step. The key part of the reconstruction step is the determination of the orientation of the segment. This is equivalent to the determination of the unit normal vector $\mathbf{n}$ to the segment. Then, the normal vector $\mathbf{n}$ and the volume fraction $C$ uniquely determine a straight line. Once the interface has been reconstructed, its motion by the underlying flow field must be modelled by a suitable algorithm [42].

\subsubsection{Implementation of surface tension}

Surface tension along an interface arises as the result of attractive forces between molecules in a fluid. In a droplet surface, the net force is radially inward, and the combined effect of the radial components of forces across the entire spherical surface is to make the surface contract, thereby increasing the pressure on the concave side of the surface. At equilibrium in this situation, the opposing pressure gradient and cohesive forces balance to form spherical drops. Surface tension acts to balance the radially inward inter-molecular attractive force with the radially outward pressure gradient across the surface. Implementation of surface tension was reviewed by Scardovelli and Zaleski [43]. Here, the continuum surface force (CSF) model proposed by Brackbill et al. [5] is employed. The addition of surface tension to the VOF method is modelled by a source term in the momentum equation.

The pressure drop across the surface depends upon the surface tension coefficient $\sigma$,

$$
\Delta p=\sigma\left(\frac{1}{R_{1}}+\frac{1}{R_{2}}\right)
$$

where $\mathrm{R}_{1}, \mathrm{R}_{2}$ are the two radii, in orthogonal directions to measure the surface curvature. In the CSF formulation, the surface curvature is computed from local gradients in the surface normal at the interface, the surface normal $\mathbf{n}$ is defined by

$$
\mathbf{n}=\nabla C_{d} \partial
$$

where $C_{\mathrm{d}}$ is the secondary phase volume fraction.

The curvature $k$ is defined in terms of the divergence of the unit normal $\hat{n}$ :

$$
k=\nabla \cdot \hat{n}=\frac{1}{|n|}\left[\left(\frac{n}{|n|} \cdot \nabla\right)|n|-(\nabla \cdot n)\right]
$$


where

$$
\hat{n}=\frac{n}{|n|}
$$

The surface tension can be written in terms of the pressure jump across the interface, which is expressed as a volume force added to the momentum equation

$$
F=2 \sigma k C_{d} \nabla C_{d}
$$

The CSF model allows for a more accurate discrete representation of surface tension without topological restrictions, and leads to surface tension forces that induce a minimum in the free surface energy configuration.

\subsubsection{Solution algorithms}

The solution algorithm involves the use of a control-volume-based technique to convert the governing equations to algebraic equations that can be solved numerically. Non-linear governing equations are linearized by an implicit scheme to produce a system of equations for the dependent variable in every computational cell. A point implicit Gauss-Seidel linear equation solver is then used, in conjunction with an algebraic multigrid (AMG) method, to solve the resultant scalar system of equations for the dependent variable in each cell.

The governing equation can be discretised in the finite volume method (FVM) as follows:

$$
\frac{(\rho \phi)_{f}{ }^{t+\Delta t}+(\rho \phi)_{f}{ }^{t}}{\Delta t} \Delta V+\sum_{f}^{N \text { faces }} \rho_{f} \phi_{f} V_{f} A_{f}=\sum_{f}^{N \text { faces }} \Gamma_{f}(\nabla \phi)_{n} A_{f}+S_{\phi} \Delta V
$$

where $N_{\text {faces }}$ is the number of faces enclosing a cell

$\phi_{f}$ is the value of $\phi$ convected through face $f$

$A_{f}$ is the area of face $f,|A|\left(=\left|A_{x} \hat{i}+A_{y} \hat{j}\right|\right.$ in 2D)

$(\nabla \phi)_{n}$ is the magnitude of $\nabla \phi$ normal to face $f$

$V$ is the cell volume

$\Gamma_{\phi}$ is a diffusion coefficient for $\phi$

$S_{\phi}$ is a source of $\phi$ per unit volume 
A linearized form of equation (13) can be written as

$$
a_{p} \phi+\sum_{n b} a_{n b} \phi_{n b}=b
$$

where the subscript $n b$ refers to neighbour cells, and $a_{p}$ and $a_{n b}$ are the linearized coefficients for $\phi$ and $\phi_{n b}$.

The pressure-velocity coupling is achieved by using the pressure-implicit with splitting of operators (PISO) scheme [39]. Since body forces (such as surface tension) are to be considered in the calculation, a special treatment (body-force-weighted scheme) is adopted to deal with the equilibrium of the body force and pressure gradient terms in the momentum equation. This induces an extra correction in the source term of the pressure and face flux rate equations, and the flow generally achieves a realistic pressure field very early in the iterative process. The standard $k-\varepsilon$ turbulence model is employed for turbulence-imposed flows.

The computational domain in our problem requires very refined grids, because of the need to capture the drop formation and breakup at a very fine scale. An efficient solution is provided by the algebraic multigrid approach, which accelerates the convergence of the solver by computing corrections on a series of coarse grid levels when the domain contains a large number of control volumes.

\section{Numerical experiments}

\subsection{Moving interface topologies}

The moving interface cases based on the test symbol were set up to estimate the topology of the interface during time integrations. The test symbol is initially assumed to be a fluid (water) in air, which then falls into a shallow pool due to the force of gravity. The computational domain is calculated on a micro-scale domain $1.6 \times 0.4$, grid size $64 \times 256$ as depicted in Fig.5, computed by the PLIC interface reconstruction schemes. Contours of velocity magnitude and static pressure clearly show the falling, deforming and merging processes. 


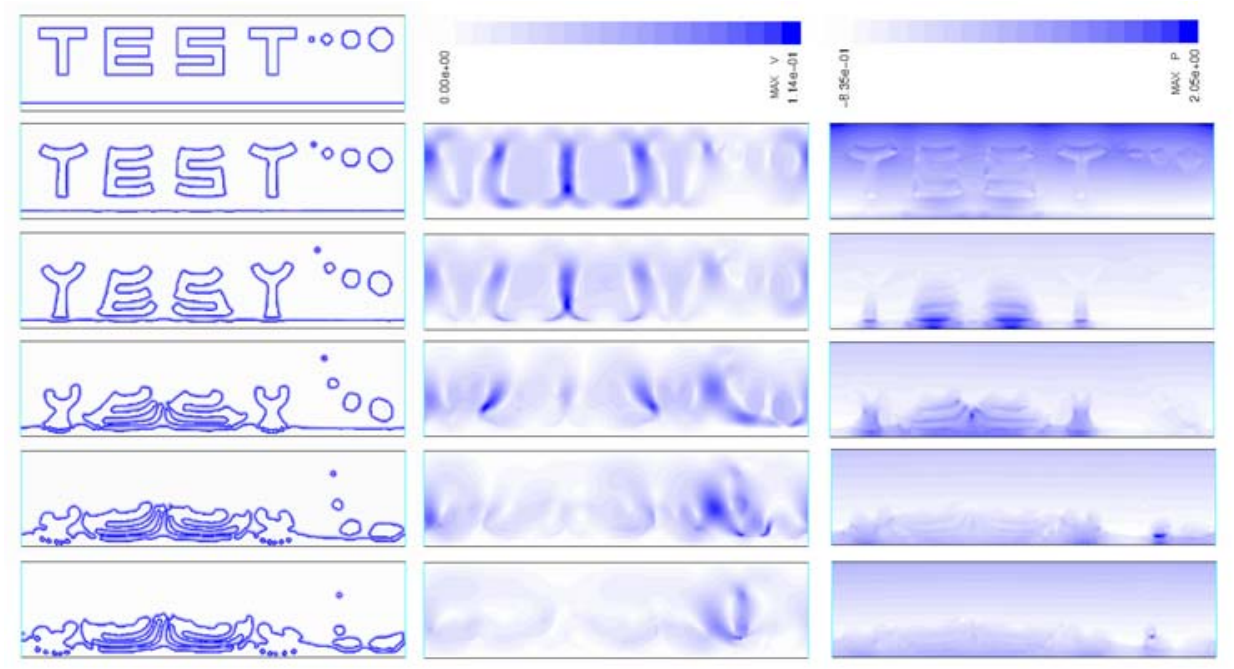

Fig. 5 Simulation results for test symbol falling into a pool in the micro-scale at time steps: $\mathrm{t}=0.0 \mathrm{~s}, 0.004 \mathrm{~s}, 0.006 \mathrm{~s}, 0.008,0.010$ and $0.012 \mathrm{~s}$. Domain size is $1.6 \times 0.4$. From left: contour of phase interface, velocity magnitude $(\mathrm{m} / \mathrm{s})$, and static pressure (Pascal).

\subsection{Rayleigh-Taylor-Instability}

The nonlinear development of Rayleigh-Taylor instability is used to investigate the interface topology and numerical convergence since this is a widely used test for validation of the VOF method $[3,5,40,44,45,46]$. In this problem, an air/helium system in a rectangular $0.01 \times 0.04 \mathrm{~m}$ domain is computed to time $0.118 \mathrm{~s}$. Grid size is $32 \times 128$. The interface is initially a sine wave with amplitude $0.5 \mathrm{~mm}$.

The results are compared with those of a high-order solver for the time integration (LVIRA) [44] for the same mesh size (Fig. 6). Similar interface propagation is achieved though there is a slight delay in the development of the mushroom cap. The reconstructed interface retains all the small-scale features of the flow.

The Rayleigh-Taylor instability problem is then further propagated to time $=0.2 \mathrm{~s}$. As shown in Fig. 7, the interface development is tracked properly and detailed interface features are clearly produced. It is also noted that the characteristics of splash are more detailed with the adaptive grid $64 \times 256$ than with the grid $32 \times 128$. 


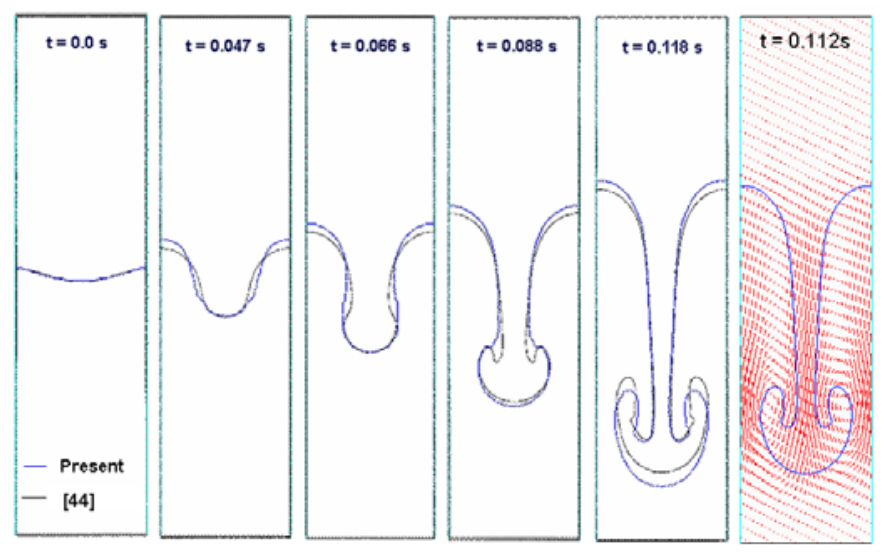

Fig. 6 Comparison of the development of the interface at different time steps with [44] and velocity vector fields at time $\mathrm{t}=0.112 \mathrm{~s}$.

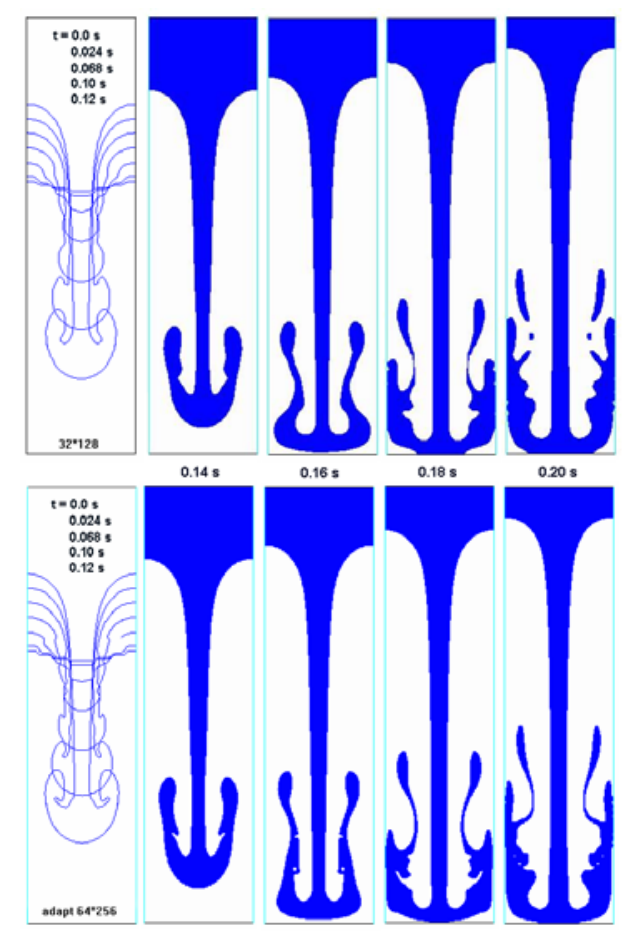

Fig. 7 Comparison of the topologies of the Rayleigh-Taylor unstable interface with different grid solutions for the interface propagation from $\mathrm{t}=0$ to 0.12 and 0.14 to $0.2 \mathrm{~s}$.

\section{Simulation of immiscible liquid alloy flows}

Here, the essential micro-mechanism of immiscible $\mathrm{Pb}-\mathrm{Zn}$ liquid alloys in rheomixing process is presented. The thermophysical properties of immiscible metallic $\mathrm{Pb}-\mathrm{Zn}$ binary alloys are taken from [47], while phase equilibrium data of $\mathrm{Zn}$ $\mathrm{Pb}$ binary alloys are taken from [48]. Shear rate is estimated by the equation $\dot{\gamma}=2 n \pi$ $\left(r_{s} / \delta-1\right)$, where $r_{s}$ is the screw radius, $n$ is the screw rotation speed and $\delta$ is the gap between barrel and screw surface [49]. Simulations are conducted on simplified flow 
fields under shear-induced force, which was implemented as moving boundaries in the computational domain coupled with imposed initial flow field condition. The breakup scale factors (BSF) $K_{n}^{t} \sim f\left(L_{d}, L_{p}, t\right)\left(L_{d}, L_{p}\right.$ - characteristic length scales of daughter droplet and parent drop, respectively) are proposed here to measure the size of the daughter drop with $t=\xi, t=\psi, t=\zeta$ the time for first daughter drop formation, full breakup up of parent drop, and well distribution of daughter droplets, respectively. The rotation speed for the experimental work in the BCAST laboratory was set at $800 \mathrm{rpm}$. The status of the immiscible alloy liquid during the twin-screw processing is controlled by a temperature control system which ensures a proper viscosity of the matrix phase from start to finish.

\subsection{Overview of immiscible liquid alloy flow in rheomixing process}

The deformations of $\mathrm{Pb}$ metallic drops in the rheomixing process are evaluated in simplified computational domains. The liquid immiscible metallic $\mathrm{Pb}$ drops break up into small droplets in shear-induced flow, with small daughter drops forming in areas of high local shear. The shear rate in the twin-screw rheomixing process is distributed in the flow field with an area of high shear rate located between the flank top of the screw and barrel wall, as well as an area located near the tip of two flights. These are defined as one-sided shear and two-sided shear imposed flows, with a low shear rate area in the middle of the screw channel.

The deformation of a $\mathrm{Pb}$ metallic drop in these areas is quite different. The $\mathrm{Pb}$ metallic drop is given an initial radius $r_{d}=1$, and the initial shear rate was imposed on the flow field according to the operating condition of the rheomixing process.

The breakup scale factor (BSF) $K_{\max }^{t}$ is defined as the ratio of the largest size of daughter drop to parent drop diameter at each time interval. $K_{\max }^{t}=\left.r_{d d \max }\right|_{t=i} / D_{d}$, $r_{d d m a x}$ denotes the largest daughter drop size, $D_{d}$ denotes the diameter of the parent drop.

The deformation of a $\mathrm{Pb}$ metallic drop in one-sided shear flow is shown in Fig.8 case 1. A sharp elongative end pinching was formed on the shear-induced side; consequently, the first daughter drop formed during breakup has quite a small radius. Later daughter drop radii are much bigger than the first few ones, and have a long rice shape, not the near spherical shape that the first daughter drop has. 
For two-sided shear-induced flows, results of the simulations are similar to case 1. Daughter drops were born on both sides as shown in Fig.8-case 2 and case 3. The drop full breakup into droplets is also easier than in one-sided shear flow, as the drop was much more elongated. A rapid shear-induced flow can lead to the quicker breakup of $\mathrm{Pb}$ metallic drops into finer droplets.

Comparison of the maximum size of daughter droplets during shearing time in laminar flow for case 4 and case 5 is shown in Fig.9. The droplet sizes are more uniform in a matrix phase of high viscosity, and there is less coalescence.

\begin{tabular}{|c|c|c|c|}
\hline$t=0.0 \mathrm{~ms}$ & $\mathrm{t}=5.0 \mathrm{~ms} \longrightarrow$ & $\infty$ & \\
\hline$t=2.0 \mathrm{~ms}$ & $\mathrm{t}=7.2 \mathrm{~ms}$ & $\infty \infty \infty=0, \cdots=$. & \\
\hline$t=2.8 \mathrm{~ms}$ & $\mathrm{t}=11.8 \mathrm{~ms}$ & $-\infty, \infty=0, \cdots$ & $\circ .0 .00$ \\
\hline \multicolumn{4}{|c|}{ case 1} \\
\hline$t=0.0 \mathrm{~ms}$ & $\mathrm{t}=4.0 \mathrm{~ms}$ & - & $0 \infty$. \\
\hline $\mathrm{t}=1.4 \mathrm{~ms}$ & $\mathrm{t}=5.0 \mathrm{~ms} \quad \ldots-\cdots$ & $-\infty=\infty-\infty$ & $\infty 000000$ \\
\hline $\mathrm{t}=2.2 \mathrm{~ms}$ & $\mathrm{t}=6.0 \mathrm{~ms}$ & $--1-2---\cdot$ & 000000000. \\
\hline \multicolumn{4}{|c|}{ case 2} \\
\hline $\mathrm{t}=0.0 \mathrm{~ms}$ & $\mathrm{t}=3.8 \mathrm{~ms}$ & D. & \\
\hline$t=2.0 \mathrm{~ms}$ & $\mathrm{t}=5.0 \mathrm{~ms}$ & & $00 \infty$ \\
\hline $\mathrm{t}=2.8 \mathrm{~ms}$ & $\mathrm{t}=6.0 \mathrm{~ms} \quad-\infty$ & $+\infty=\infty-\infty$ & $\infty \infty \infty$ \\
\hline
\end{tabular}

Fig. 8 A sequence of deformation leading to breakup of a $\mathrm{Pb}$ metallic drop and evolution of rupturing behaviour of a $\mathrm{Pb}$ metallic drop in shear-induced flow, grid $128 \times 32$, domain $16 \times 4$, case 1 and case 2 are for $\lambda=1, \mathrm{Ca}=3.2$ for one side shearinduced flow and double side shear-induced flow respectively and with enhanced initial shear rate near wall. Case 3 is for $\mathrm{Ca}=1.17, \mathrm{Re}=0.0$ initial flow field. 


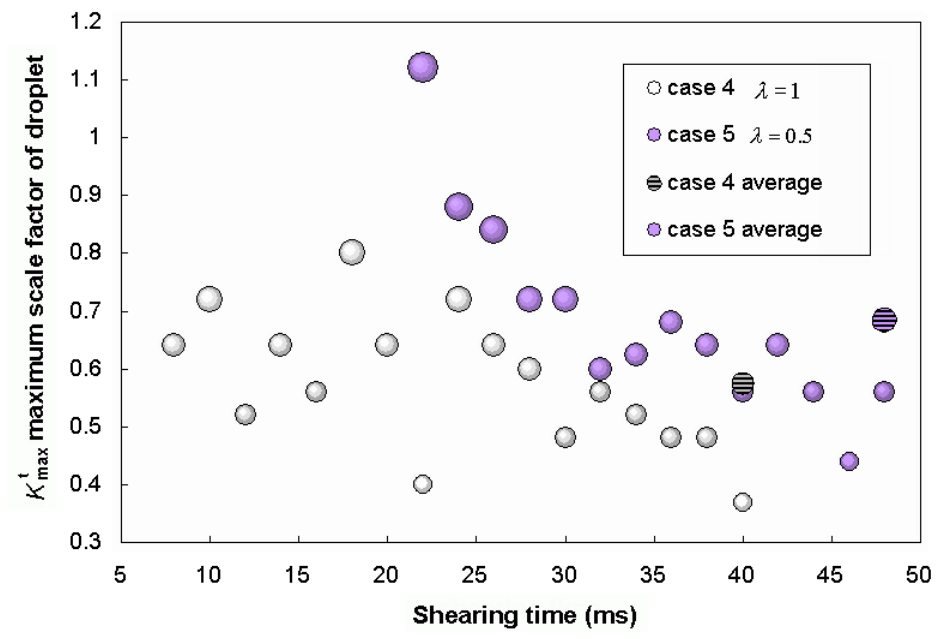

Fig. 9 Comparison of maximum size of daughter droplet during shearing time in laminar flow for case 4 and case 5. Shearing time indicated in the chart starts from the time $t=\psi$.

\subsection{Morphology of droplets flow in TSE channel and gap}

The twin-screw used in the rheomixing process has a $16 \mathrm{~mm}$ diameter at tip and 3 $\mathrm{mm}$ groove with a special profile to achieve high shear rate and enhance the positive displacement pumping action. The maximum rotation speed of the screw is designed at $1000 \mathrm{rpm}$, which corresponds to a shear rate at $4082 \mathrm{~s}^{-1}$ in the gap between the tip of the screw flight and the barrel [1].

The deformation is slightly different from that in the two domains corresponding to shear-induced flow in the TSE channel and TSE gap. Due to micro-scale drop radius and the effect of surface tension that lead to the drop maintaining a round shape, the sharp ending deformation does not appear in the micro-scale domain. The morphology of droplet dispersion at a later stage is relative similar in both domain sizes as depicted in Fig.10, and the qualitative analysis of droplet dispersion on both domain sizes will not produce significant differences.
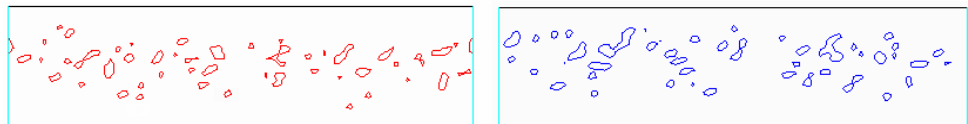

Fig. 10 Comparison of morphology of droplet distribution in TSE channel (left) and gap (right). 


\subsection{Comparison with experimental results}

The numerical evolution of droplet dispersion is compared with an experimental image [33] for the distribution of maximum droplet sizes above $32 \mu \mathrm{m}$ of diameter in Fig.11, as the main concern in rheomixing is the maximum droplet size. It is observed that the numerical results show good qualitative agreement with the experimental image. The quantitative difference is shown in the graph of Fig.11. The distribution of smaller droplet sizes is difficult to obtain due to: (1) the finest droplets were produced by the reorientation process of TSE, however the shearing in the computational simulation is non-reorientating; (2) the limitation of grids for the computation; the grid would need to increase hundreds of times in order to capture a $20 \mu \mathrm{m}$ droplet. The present numerical simulation results show that further numerical predictions can be explored to provide a deeper insight into the microstructure mechanism of the rheomixing process.

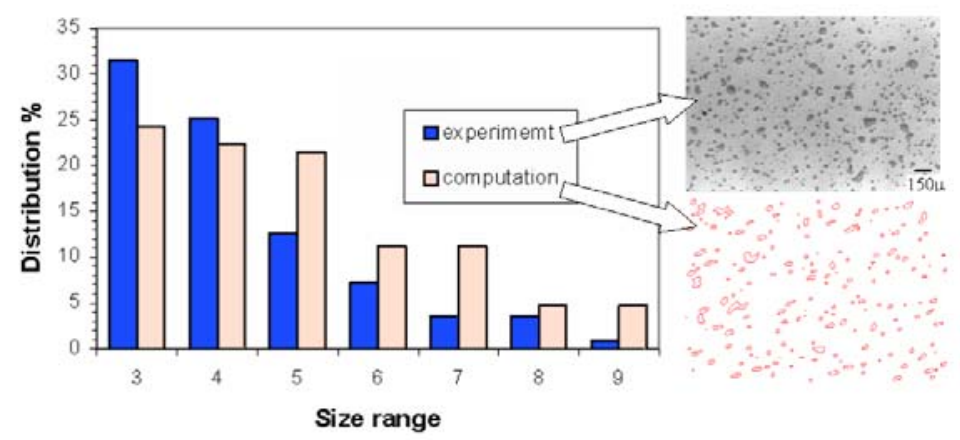

Fog. 11 Comparison of the droplet size distribution with experimental image data.

\subsection{Optimisation design of rheomixing process}

\section{Screw configuration}

Generally, the right hand screw transports materials, the left hand screw controls the pressure field, while the kneading disc melts and mixes materials. The flow regime is envisaged as being composed of three distinct types of flow: pressure flow, net flow and drag flow. These produce different shear rate profiles. Shear rate profiles influence drop formation as illustrated in Fig.8. Therefore, a proper combination of right-hand screws, left-hand screw and kneading disc is a critical factor to optimise 
shear rate profiles and enhance turbulence. This means that the TSE configuration is the most important factor to achieve the folding and reorientation actions; the continuous reorientation in the TSE process could produce fine droplets and reach best mixing efficiency.

\section{Operating condition}

a. Temperature - Control of the viscosity of the matrix phase by temperature is important because it will balance the Stokes and Marangoni motion of droplets (that cause sedimentation and migration, resulting in stratification and segregation in the microstructure, respectively), which are the main obstacles to the industrialization of immiscible alloy casting.

b. Rotation speed - The shear rate is dependent on rotation speed as mentioned in Section 6 . The shear rate will vary for different screw parameters. This includes the screw size: pitch, left-handed helix, right-handed helix, size of kneading discs: angle, crest width as well as the number of screws and discs, and their arrangement. The most important phenomenum in the rheomixing process by a twin-screw extruder is enhanced shear rate; the local shear rate could be higher than $3600 \mathrm{~s}^{-1}$. This allows dramatic results in the dispersion and refinement of $\mathrm{Pb}$ liquid droplets in the $\mathrm{Zn}$ matrix phase, leading to fine droplets and uniform dispersion.

\section{Optimisation procedure}

According to the results of numerical investigations, possible suggestions for optimising the rheomixing process are proposed in following procedure: start shearing immiscible metallic binary alloys under enhanced turbulence and temperature above $\mathrm{T}_{\mathrm{m}}$. Both phases have the same viscosity value, which might cause fast breakup and fine droplets, shorten the time for a full breakup, reducing power consumption. If shearing remains at temperature $\mathrm{T}_{\mathrm{m}}$, this will result in spherical droplets as the viscosity of the matrix phase is increased. Droplets will also be dispersed stably in thick matrix phase and will be suspended homogenously in the matrix phase.

\section{Conclusions}

The numerical simulation of the essential microscopic mechanisms of a prototypical rheomixing process is useful for the optimisation of the rheomixing process. The simulation model can be used to obtain an insight into shearing time, 
viscosity and shear force, thus providing a guide to the operating condition of rheomixing process in order to reduce trial and error experiments for optimising parameters. It is concluded that rheomixing by a twin-screw extruder (TSE) was successfully developed for casting immiscible engineering alloys due to its unique characteristics of reorientation and surface renewal.

\section{Acknowledgements}

We acknowledge support from EPSRC grant GN/N14033, Glacier Vanderville Ltd, Ford Motor Co. Ltd, Prism Ltd, QinetiQ Ltd. and the Mechanical Engineering Department at Brunel University. We are also grateful to researchers in CFD group and BCAST (Brunel Centre for Advanced Solidification Technology) for helpful discussions on numerical approaches and the TSE rheomixing casting process.

\section{References}

[1] Z. Fan, S. Ji, J. Zhang: Materials Science and Technology 17: 838 (2001)

[2] L. Ratke, S Diefenbach: Material Science and Engineering RI5: 263 (1995)

[3] D.L. Youngs: Time-dependent multi-material flow with large fluid distortion, In:. K.W. Morton, M.J. Baines (eds) Numerical Methods for Fluid Dynamics (Academic Press, New York 1982) pp. 273

[4] C.W. Hirt, B.D. Nichols: J Comput Phys 39: 201 (1981)

[5] J.U. Brackbill, D.B. Kothe, C. Zemach: J. Comput. Phys. 100: 335 (1992)

[6] ESA: The international space station microgravity: a tool for industrial research. European Space Agency; BR-136 (1998)

[7] W. Zeng, J. Guo, N. Chen, J. Guo: Calphad 21: 289 (1997)

[8] Z. Zhang, Z. Su, Z. Wu, N. Chen, R. Peng: Calphad 22: 313-322 (1998)

[9] Y. Plevachuk, V. Didoukh, B. Sokolovskii: Journal of Non-Crystalline Solids 250-252: 325 (1999)

[10] J.H. Shim, Lee HN, H.P. Ha, Y.W. Cho, E.P. Yoon: Journal of Alloys and Compounds 327: 270 (2001)

[11] J. Connolly: Phase diagram principle and computations. In: Pressure and Temperature Evolution of Organicbelts, Lecture notes of the V summer school, The University of Siena, The Italian national Research Council, 203-220 (1992)

[12] P.M. Smith, J.W. Elmer: Acta mater. 44: 4217 (1996)

[13] C. Gueneau, V. Dauvois, P. Pérodeaud, C. Gonella, O. Dugne: J Nuclear Material 254: 158 (1998)

[14] L. Ratke, J. Alkemper: Advances in Colloid and Interface Science 58: 151 (1995)

[15] .Z. Zhao, S. Drees, L. Ratke: . Materials Science and Engineering A 282: 262 (2000)

[16] N.O. Young, J.S. Goldstein, M.J. Block: J. Fluid Mech. 6: 350 (1959), cited in [19].

[17] R.J. Naumann: Journal of Crystal Growth 154: 156 (1995)

[18] Z. Zapryanov, S. Tabakova: Dynamics of Bubbles, Drops and Particles The Netherlands: Kluwer Academic Publishers (1999)

[19] X.Y. Lu, C.D. Cao, B. Wei: Materials Science and Engineering-A 313: 198 (2001)

[20] B. Nestler, A.A. Wheeler, L. Ratke, C. Stöcker: Physica D: Nonlinear Phenomena 141: 133 (2000)

[21] N Rudraiah, P.G. Siddheshwar: Aerosp Sci Technnol 4: 517 (2000)

[22] T. Hibiya, S. Nakamura, T. Azami, M. Sumiji, N. Imaishi, K. Mukai, K. Onuma, S.I. Yoda: Acta Astronautica 48: 71 (2001)

[23] K. Takagi, M. Otaka, H. Natsui, T. Arai, S. Yoda, Z. Yuan , K. Mukai, S. Yasuhiro, N. Imaishi: Journal of Crystal Growth 3: 399 (2001)

[24] L. Ratke: Materials Science and Engineering A203: 399 (1995)

[25] L. Ratke: International Journal of Multiphase Flow 22: supplement 1, 92 (1996)

[26] M. Rhazi, A. Mir, Z. Zrikem, G. Gouesbet: International Communications in Heat and Mass Transfer 23: 345 (1996)

[27] L. Ratke, S. Diefenbach, S. Drees, J. Alkemper, B. Prinz, A. Romero, H. Ahlborn: Advances in Space Research 16: 185 (1995) 
[28] J.F. Agassant, A. Poitou: A kinematic approach to distributive mixing. In: I. Manas-Zloczower and Z. Tadmor (eds), Mixing and Compounding of Polymer (Hanser, New York 1994)

[29] C. Rauwendaal: Mixing in Polymer Processing (Marcel Dekker, New York 1991)

[30] Meijer HEH, Janssen MH. Mixing of immiscible liquids. In: I. Manas-Zloczower, Z. Tadmor (eds), Mixing and compounding of polymer (Hanser, New York 1994) p52, p85

[31] J. Jia, J.Z. Zhao, J.J. Guo, Y. Liu: Immiscible Alloys and Fabrication Technology (HIT, Harbin (China) 2002)

[32] Z. Fan: Twin-screw rheoforming technologies for semisolid processing of Mg-alloys. In: Y. Tsutsui, M. Kiuchi, K. Ichikawa (eds) Proceeding of the $7^{\text {th }}$ Advanced Semisolid Processing of Alloys and Composites Tsukuba 25-27 September 2002. Japan: NIAIST, JSTP, 671-676

[33] X. Fang, Z, Fan, S, Ji, Y, Hu: Processing of immiscible alloys by a twin-screw rheomixing process. In: Y. Tsutsui, M. Kiuchi, K. Ichikawa (eds) Proceeding of the $7^{\text {th }}$ Advanced Semisolid Processing of Alloys and Composites Tsukuba 25-27 September 2002, Japan: NIAIST, JSTP, 695-700

[34] N.P. Cheremisinoff: Polymer Mixing and Extrusion Technology (Marcel Dekker, Inc, New York 1987)

[35] T. Avalosse: Twin screw extruder and mixing tank Presentation of Polyflow s.a., Belgium: Louvain-la-Neuve (2000)

[36] S. Bakalis, M.V. Karwe: Int. J. Food Science and Technology 32: 241 (1997)

[37] K. Stüben: Journal of Computational and Applied Mathematics 128(1-2): 281 (2001)

[38] B.E. Launder, D.B. Spalding: Comput. Meth. App. Mech. Engng. 3: 267 (1974)

[39] R.I. Issa, A.D. Gosman, P. Watkins: J. Comput. Phys. 93: 388 (1991)

[40] M. Rudman: Int. J. Num. Methods Fluids 24: 671 (1997)

[41] O. Ubbink, R.I. Issa: J. Comput. Phys. 153: 26 (1999)

[42] C.S. Peskin: J. Comput. Phys. 25: 220 (1997)

[43] R. Scardovelli, S. Zaleski: Annu. Rev. Fluid Mech. 31: 567 (1999)

[44] E.G. Puckett, A.S. Almgren, J.B. Bell, D.L. Marcus, W.J. Rider: J. Comput. Phys. 130: 269 (1997)

[45] G. Černe, S. Petelin, I. Tiselj: J. Comput. Phys. 171: 776 (2001)

[46] S. Guignard, R. Marcer, V. Rey, C. Kharif, P. Fraunié: Eur. J. Mech. B/Fluids 20: 57 (2001)

[47] T. Iida, R.I.L. Guthrie: The Physical Properties of Liquid Metals (Oxford University Press, New York 1988)

[48] R. Hultgren, R.L. Orr, P.D. Anderson, K.K. Kelley: Selected Values of the Thermodynamic Properties of Binary Alloys (Metals Park, ASM 1973)

[49] C. Rauwendaal: Polymer Extrusion $3^{\text {rd }}$ rev. ed. (Hanser, New York 1994) pp.181 